\title{
Review on Speckle-Based Spectrum Analyzer
}

\author{
Yangyang WAN, Xinyu FAN*, and Zuyuan HE* \\ State Key Laboratory of Advanced Optical Communication Systems and Networks, Shanghai Jiao Tong University, \\ Shanghai 200240, China
}

Corresponding authors: Xinyu FAN and Zuyuan HE

Emails: fan.xinyu@sjtu.edu.cn and zuyuanhe@sjtu.edu.cn

\begin{abstract}
Accurate spectral measurement and wavelength determination are fundamental and vital for many fields. A compact spectrum analyzer with high performance is expected to meet the growing requirements, and speckle-based spectrum analyzer is a potential solution. The basic principle is based on using the random medium to establish a speckle-to-wavelength mapping relationship for spectrum reconstruction. This article introduces current speckle-based spectrum analyzers with different schemes and reviews recent advances in this field. Besides, some applications by using speckle-based spectrum analyzers are also introduced. Finally, the existing challenges and the future prospects of using speckle for spectrum recovery are discussed.
\end{abstract}

Keywords: Speckle; spectrum analyzer; random medium; spectroscopy

Citation: Yangyang WAN, Xinyu FAN, and Zuyuan HE, "Review on Speckle-Based Spectrum Analyzer," Photonic Sensors, 2021, 11(2): 187-202.

\section{Introduction}

Spectroscopy is an essential tool used in many fields of scientific researches, such as biological sensing [1], environmental monitoring [2, 3], astronomy [4, 5], medical applications [6, 7], and optical communication [8]. Conventional spectrometers diffract the spectral components of lightwave to different positions of the photodetector (PD) array by using a grating. The resolving power $R$ of available commercial spectrometers is usually between $10^{5}-10^{7}[9,10]$, which is defined as $R=f_{0} / \delta f$. Here, $\delta f$ is the spectral resolution at the frequency $f_{0}$. The spectral resolution of the grating-based spectrometers scales inversely with the optical path length between the PD array and the grating. With a longer optical path length, it is easier to distinguish two adjacent wavelengths which are measured by different PDs and a higher spectral resolution can be achieved. In order to achieve a high-resolution grating spectrometer, the system is therefore inevitably bulky. Motivated by vast applications, the realization of a compact spectrometer with high-performance has always been a hot research topic and researchers have developed many types of novel spectrometers without using gratings. Fourier transform (FT) spectrometer is a compact system [11], but it is still a challenge to achieve a high spectral resolution with the limited optical path difference of the Mach-Zehnder interferometer for this kind of spectrometers [12]. Moreover, the measurement rate of FT spectrometer is low due to the scanning interferometric configuration for large bandwidth applications $[13,14]$.

Recently, speckle-based spectrum analyzers have drawn the attention of researchers due to their characteristics of compactness and high-resolution. 
Lightwave which transmits through a random medium will produce a speckle pattern with random intensity distribution. The common random medium may be rough surface [15], integrating sphere [16], multimode fiber [17], and so on [18, 19]. The principle of the speckle generation is the multiple interference of scattered lightwave [16] or the interference between the guided modes [17, 20] in the random medium. In the field of optical imaging and optical communication, since the appearance of speckle corrupts the optical image or the information to be transmitted, researchers made many efforts to eliminate the speckles $[21,22]$. It is noted that the speckle is sensitive to the change of system structure and the environmental perturbation. Therefore, the unique speckle pattern can also be used for sensing applications [23]. Some works used the speckle generated by the optical fiber for sensing strain [24], temperature [25], and displacement [26], which are also named as specklegram sensors. In addition to measure the structure and environment, we can acquire some information of the incident lightwave from the speckle when the configuration is fixed. After propagating through the random medium, the spectrum and polarization information are hidden in the speckle which has a random intensity distribution. The corresponding generated speckle is varied with the incident lightwave, and we can retrieve the spectrum of the incident lightwave from the speckle pattern [27]. As one wavelength corresponds to one spatial speckle with a special intensity distribution mapping relation, it is used for spectrum recovery in the speckle-based spectrometer or wavemeter.

Typically, the charge coupled device (CCD) camera or PD array serves as the detector array to capture the two-dimensional (2D) or one-dimensional spatial speckle in this kind of spectrum analyzer. The introduction of CCD makes the systems expensive and limits the measurement rate due to the finite frame rate of the camera. Moreover, the systems with CCD are more likely influenced by environmental perturbation since they require accurate spatial calibrations of the individual components. The systems which capture onedimensional spatial speckle by using PD array can only achieve a low dynamic range (bandwidthto-resolution ratio) limited by the PD number [28]. In addition to the spatial speckle-based spectrum analyzer (SSSA), we recently proposed a novel temporal speckle-based spectrum analyzer (TSSA) by using only a single PD for detection. When lightwave transmits in a single-mode fiber (SMF), Rayleigh scattered lightwave is produced along the whole fiber [29]. Since the refractive index distribution of SMF is not uniform, Rayleigh scattered lightwave at different positions on the fiber have different optical path differences [30]. Rayleigh backscattered lightwave from different reflection points interfere with each other on the detector to produce a temporal Rayleigh speckle with a jagged appearance [31, 32]. Therefore, SMF is regarded as the random medium to generate speckle in this temporal mode and the system only needs a single PD to obtain the Rayleigh speckle. With the advantage of using a single PD, the temporal speckle configuration can realize a higher measurement rate and be more cost-effective and compact.

In this review, we focus on the recent advances in speckle-based spectrum analyzer. The article is organized as follows. In Sections 2 and 3, we introduce the basic principles of SSSA and TSSA, respectively. The definitions of resolution, bandwidth and measurement rate of each system, and methods for performance improvement are discussed in the corresponding sections. Section 4 introduces some existing applications based on speckle-based spectrometer or wavemeter. Section 5 discusses the open challenges in this field. Finally, the conclusion and outlook on the speckle-based spectrum analyzer are discussed in Section 6 .

\section{Spatial speckle-based spectrum analyzer (SSSA)}

The conventional grating-based spectrometers 
make use of the one-to-one spectral-spatial mapping to achieve spectrum measurement. This spectralspatial mapping relation is indispensable for wavelength demultiplexer, but not so essential for spectrum analyzer. The spectrometers or wavemeters have been demonstrated based on another mapping relation that one spectral input corresponds to one pattern of spatial speckle $[27,16]$.

\subsection{Principle and configuration}

The basic configuration of SSSA is illustrated in Fig. 1(a). The system only consists of a random medium and a CCD camera. The lightwave is transmitted through the random medium to produce the speckle detected by the camera. Distinct speckles are generated by lightwave with different wavelengths. Before measurement, a database containing various speckles of different singlewavelength lightwave needs to be built. Once the database is established, we can recover the spectrum information of the incident lightwave from the database. Although the basic principle is similar, the performance and characteristics of SSSAs are slightly different due to the use of different types of random mediums. A novel and interesting random medium is multimode fiber (MMF). In the MMF-based spectrometers as shown in Fig. 1(b), the speckle is produced by the interference between multiple lightwave guided modes in the MMF [33]. Integrating sphere and rough surface are other kinds of common random mediums [16, 34-36]. After the incident lightwave passes through these random mediums, the speckle is formed by the interference between the multiple scattered lightwave. The experimental setup of the integrating sphere-based wavemeter is shown in Fig. 1(c). Most random mediums used for speckle generation are artificial and require high-precision nano-fabrications. An interesting work has demonstrated that a natural pearl can also be used to obtain random speckle pattern for spectrum recovery as shown in Fig. 1(d) [37]. Since the natural structure of the pearl has an extremely large number of thin layers which is comparable to the artificial production, the pearl spectrometer achieves a spectral resolution of $7.4 \mathrm{~nm}$ with the system bandwidth of $250 \mathrm{~nm}$.

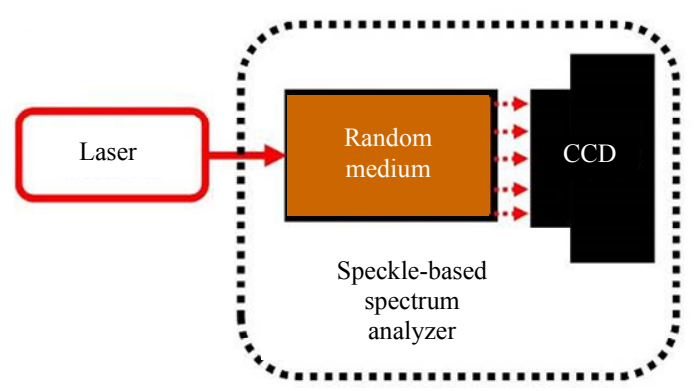

(a)

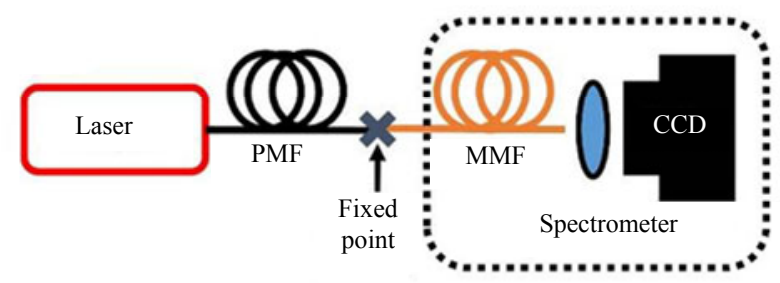

(b)

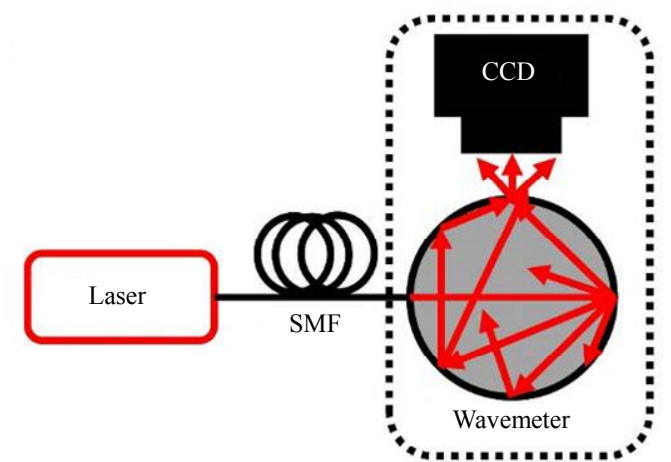

(c)

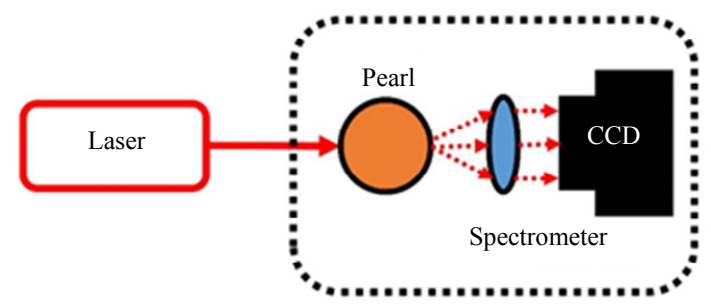

(d)

Fig. 1 Schematic of SSSA with different random media: (a) the basic configuration of spatial speckle-based spectrum analyzer, (b) the schematic of the multi-mode fiber spectrometer setup [PMF, polarization-maintaining fiber; MMF, multi-mode fiber], (c) the experimental setup of the integrating sphere-based wavemeter. SMF, single-mode fiber, and (d) the experimental setup of the spectrometer based on a pearl.

The filter array can also be regarded as a random medium to generate unique speckle pattern for spectrum determination of the incident lightwave as 
shown in Fig. 2. In a conventional filter array spectrometer (top path in Fig. 2), the filter array of bandpass filters samples the incident spectrum uniformly. The spectrum can be read directly from PD array. Since the random filter array has a complex and variable spectral responsivity, the incident lightwave is randomly sampled in the frequency domain [38]. Therefore, a random speckle is detected by the PD array in the configuration of the random filter array (bottom path in Fig. 2) and can be reconstructed to acquire the spectrum of incident lightwave. As long as the structure has a complex and variable spectral response, it can be used as a spectrometer. The speckle spectrometers based on encoded etalon array [39], colloidal quantum dots (CQD) filters [40], nanowire [41], photonic crystal cavity array [42] or slabs [43], and 2D thin-film filter-array [44] have been proposed.

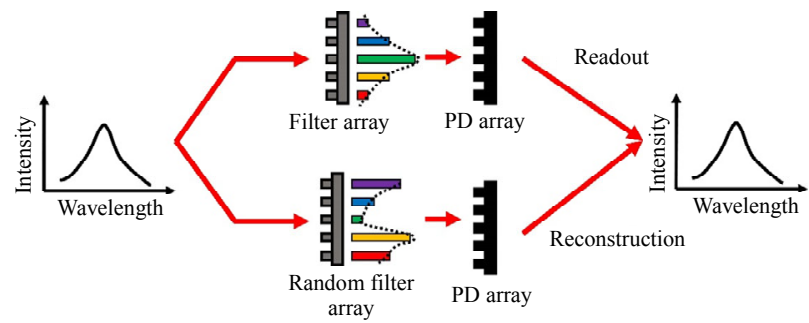

Fig. 2 Schematic of filter array based spectrometers. The top path illustrates the principle of a conventional filter array spectrometer. The bottom path illustrates the principle of random filter array spectrometer.

In order to use the random medium mentioned above to realize spectrum analyzers, a calibration process is necessary to determine the characteristics of the random medium. The lightwave intensity at the output of the random medium can be expressed as

$$
I(r, \theta)=\int T(r, \theta, \lambda) S(\lambda) \mathrm{d} \lambda
$$

where $I(r, \theta)$ is the intensity at the circle coordinates of $(r, \theta), S(\lambda)$ is the spectrum information of the incident lightwave, and $T(r, \theta, \lambda)$ is the transmission matrix. Transmission matrix demodulation algorithm is a commonly used simple and effective method for speckle-based spectrometer [45]. To determine the transmission matrix experimentally, it is necessary to discretize (1). Then the relation between the spatial intensity distribution $\mathbf{I}$ of the speckle and the transmission matrix $\mathbf{T}$ after the discretization is expressed as

$$
\left(\begin{array}{ccc}
\mathbf{T}_{11} & \ldots & \mathbf{T}_{m 1} \\
\vdots & \ddots & \vdots \\
\mathbf{T}_{1 n} & \cdots & \mathbf{T}_{m n}
\end{array}\right)\left[\begin{array}{c}
\mathbf{S}_{1} \\
\vdots \\
\mathbf{S}_{m}
\end{array}\right]=\left[\begin{array}{c}
\mathbf{I}_{1} \\
\vdots \\
\mathbf{I}_{n}
\end{array}\right]
$$

where $m$ and $n$ are the numbers of spectral and spatial channels, respectively. During the calibration process, we record the speckle corresponding to different wavelengths by changing the wavelengths of the input lightwave. After reshaping the recorded reference speckle into one dimension, it is used to determine the column of the transmission matrix $\mathbf{T}$. Once $\mathbf{T}$ is determined, the spectrum is demodulated by the following relation:

$$
\mathbf{S}=\mathbf{T}^{-1} \mathbf{I} \text {. }
$$

To avoid (3) becoming ill-conditioned, the interval of spectral channel in the transmission matrix $\mathbf{T}$ should be not larger than the spectral resolution. Note that the polarization state of the incident lightwave and the state of the structure are supposed to be unchanged after the calibration process.

\subsection{Spectral resolution}

For speckle-based spectrometers, spectral correlation function is widely adopted to determine the spectral resolution of the system. Spatial speckles at different wavelengths are recorded and the spectral correlation function is calculated by

$$
C(\Delta \lambda)=\frac{\langle I(\lambda) I(\lambda+\Delta \lambda)\rangle}{\langle I(\lambda)\rangle\langle I(\lambda+\Delta \lambda)\rangle}-1
$$

where $I(\lambda)$ is the intensity of the speckle at the wavelength $\lambda$, and $\langle\cdots\rangle$ means the average over all wavelengths. The wavelength shift $\delta \lambda$ is defined as spectral resolution and satisfy the relation $C(\delta \lambda)=C(0) / 2$. When the wavelength changes $\delta \lambda$, it can be considered that the corresponding speckles are uncorrelated. So spectral resolution is defined to be the half width at half maximum (HWHM) of its wavelengths correlation peak. Since 
a higher spectral resolution means a more precise spectrum measurement, many efforts have been devoted to improve this parameter.

For MMF-based spectrometer, researchers have thoroughly investigated the factors which influence the system resolution $[33,46]$. Using a longer MMF is an intuitive way to achieve a higher resolution by increasing the optical path difference. A $0.4 \mathrm{~nm}$ spectral resolution is achieved by using a $1 \mathrm{~m} \mathrm{MMF}$, while the resolution of $8 \mathrm{pm}$ is realized with a 20-m-long fiber [33]. By using a $100 \mathrm{~m} \mathrm{MMF,} \mathrm{the}$ MMF-based spectrometer realized a $1 \mathrm{pm}$ spectral resolution [47]. The numerical aperture (NA) of MMF also has an effect on the resolution. Researchers reveal that the resolution of MMF-based spectrometers scales linearly with $1 / L$ or $1 / N A^{2}$ [33]. Moreover, they also demonstrate that a higher resolution of MMF-based spectrometer can be achieved when more guided modes are excited in the fiber [33]. It has been shown that a larger offset displacement between the lightwave source and MMF can achieve a higher resolution by exciting more guiding modes [48]. Besides choosing suitable structure parameters for MMF, a mechanism of using an in-line polarization rotator to perform polarization modulation is another effective method to improve the resolution [49]. Moreover, an appropriate demodulation algorithm is very important for the resolution improvement. After adopting the principal component analysis (PCA), the resolution of speckle-based wavemeter with an 18-cm-long MMF can be improved from $620 \mathrm{pm}$ to attometer resolution [50]. Although the PCA algorithm provides an eight orders of magnitude resolution improvement, it is commonly designed to recover the spectrum with only a single wavelength. To overcome this problem, a work has been performed to demonstrate that PCA can also be used to deal with the sparse multiple wavelength measurement with two dynamic lasers measured simultaneously in the experiment [51].

As for the wavemeter based on integrating sphere, the method of extending the sphere diameter can increase the mean free path within the sphere and improve the spectral resolution. The system with $10 \mathrm{~cm}$ sphere diameter is demonstrated to have a $0.3 \mathrm{fm}$ spectral resolution after using PCA algorithm [16]. In addition to the PCA algorithm, deep learning is introduced for speckle-based wavemeter to improve the resolution. Convolutional neural network $(\mathrm{CNN})$ is a typical tool which may be optimized for spectrum recovery from the speckle in the reference [52]. With the powerful helps of $\mathrm{CNN}$, the system experimentally distinguishes the wavelengths with an interval of $2 \mathrm{am}$. All the demodulation algorithms mentioned above, i.e., transmission matrix method, PCA and CNN, have a calibration process before the measurement process to record speckles for their references. Although higher spectral resolution may be expected by choosing suitable structure and demodulation algorithm, the realizable spectral resolution is limited by the resolution of the tunable laser for the generation of reference speckles.

\subsection{System bandwidth}

The system bandwidth of spectrum analyzer determines the measurable spectrum range. A broader bandwidth implies more potential applications. For speckle-based spectrum analyzer, the system bandwidth is the transmission window of the random medium in theory. However, most systems are suffering from the trade-off relation between the spectral resolution and the system bandwidth. The cause of this resolution-bandwidth restriction varies with the implementations.

From (2), it is clear that the unique solution can not be obtained when the number of spatial channels $n$ is less than the number of spectral channels $m$. Therefore, the number of detectors is a limitation factor for bandwidth. Generally, the bandwidth restriction caused by the number of detectors occurs in the on-chip speckle-based spectrometers [28]. To enlarge the system bandwidth, a scheme with the capability to change the input launch condition is proposed to obtain a broad bandwidth [53, 54]. A 
single-mode input switching matrix configuration is used to excite distinct modes in a compact silicon multimode waveguide spectrometer for bandwidth enlargement. The usable bandwidth range increases from $22 \mathrm{GHz}$ to $250 \mathrm{GHz}$ by adopting this multiple input method [53]. Another integrated speckle-based spectrometer based on multimode waveguide achieves an enlarged bandwidth of $5.8 \mathrm{~nm}$ with the spectral resolution of $0.16 \mathrm{~nm}$ by controlling the mode of the incident lightwave via photonic lantern [54]. Different launch conditions will generate independent speckles, and an $(N-1)^{2}$ times bandwidth improvement can be realized with $N$ input waveguides. Besides, the method of applying compressive sensing is another effective method to realize a larger bandwidth for sparse spectra reconstruction [55].

For the MMF-based spectrometer, the total number of guided modes in MMF is the main factor that restricts the system bandwidth. This resolution-bandwidth trade-off relation is demonstrated in [47], where a $100 \mathrm{~nm}$ bandwidth is achieved with a $0.4 \mathrm{~nm}$ spectral resolution and a $1 \mathrm{~nm}$ bandwidth is achieved with an $8 \mathrm{pm}$ spectral resolution. A scheme to integrate five MMFs via a wavelength division multiplexer is proposed to achieve a $100 \mathrm{~nm}$ bandwidth with $0.03 \mathrm{~nm}$ spectral resolution at the wavelength of $1500 \mathrm{~nm}$ [56]. Integrating MMF with a multi-core fiber through a space-division multiplexing is another bandwidth enlarging method [57]. Three cores of a seven-core fiber are used to achieve a $30 \mathrm{~nm}$ bandwidth with $0.02 \mathrm{~nm}$ spectral resolution at the wavelength of $1550 \mathrm{~nm}$.

Both the transmission matrix method and PCA are limited by the number of detectors and only work in a finite bandwidth. The bandwidth of integrating sphere-based wavemeter which adopts PCA as demodulation algorithm improves with the increased image size [16]. The bandwidths of $16 \mathrm{fm}$ and $60 \mathrm{fm}$ are achieved at the speckle image size of 256 pixels $\times 16$ pixels and 256 pixels $\times 128$ pixels, respectively. The deep learning method is demonstrated as an ideal demodulation algorithm to overcome the resolution-bandwidth trade-off relation for speckle-based wavemeter [52]. In the experiment, a single CNN is used to extract wavelength information from the speckle over an operating bandwidth from $488 \mathrm{~nm}$ to $976 \mathrm{~nm}$ with the precision of attometre-scale.

\subsection{Measurement rate}

Rapid spectrum measurement is vital for laser wavelength stabilization, biological sensing, environmental monitoring, and so on. The measurement rate of the conventional grating spectrometer and FT spectrometer is difficult to reach $\mathrm{kHz}$ due to the existence of the scanning configuration [9, 13]. Although the speckle is obtained by a single-shot acquisition, the measurement rate of the speckle-based spectrum analyzer is limited by the camera response. By using a high-performance camera with a fast frame rate, the system can achieve a rapid measurement rate. In [50], 5000 speckles with the size of 8 pixels $\times$ 1024 pixels are detected within $3 \mu$ s at $48 \mathrm{kHz}$ frame rate. When using the wavemeter for laser stabilization, the maximum lock update rate only achieves $200 \mathrm{~Hz}$ by using PCA in [16]. The Poincare descriptors algorithm is proposed to accomplish the wavelength determination and a high-speed real-time measurement is achieved with the measurement rate of $>4 \mathrm{kHz}$ [35].

\subsection{System size}

One of the significant advantages of specklebased spectrum analyzer is that random mediums can be designed with a compact size. The implementations are consisted of a random medium and a camera, and the size of random medium usually does not exceed decimeter level. Note that the long MMF which has meter-scale or even reach 100 meter used for MMF based spectrometer can be coiled to a small volume [47]. Recently, an extremely compact speckle-based spectrometer with a millimetre-scale size is achieved by utilizing a multimode fiber taper as the random medium [20]. 
In order to obtain a micro spectrometer, the chip-scale speckle-based spectrometer has been a hot research topic in recent years. On a silicon on-insulator wafer, a speckle-based spectrometer with a size of $50 \mu \mathrm{m} \times 100 \mu \mathrm{m}$ achieves a resolution of $0.75 \mathrm{~nm}$ and a bandwidth of $25 \mathrm{~nm}$ [28]. The similar structure is also implemented on the silicon nitride platform to cover the bandwidth from the visible wavelengths to the telecom wavelength regime [58-60]. On-chip spectrometers are also subject to the trade-off relation of size and resolution. The silicon microstrip waveguide with a size of $4.85 \mu \mathrm{m} \times 220 \mathrm{~nm} \times 4 \mathrm{~cm}$ is fabricated to be a spectrometer with a resolution of $0.016 \mathrm{~nm}$ at the wavelength of $1550 \mathrm{~nm}$ [53]. However, even with a bandwidth enhancement scheme, the multimode waveguide spectrometer only has a bandwidth of $2 \mathrm{~nm}$.

The size of the SSSA based on random filter array can also reaches the chip-scale. The photonic crystal cavity array consists of $3 \times 3$ cavities with a pitch of $3 \mu \mathrm{m}$ used to achieve a $0.3 \mathrm{~nm}$ spectral resolution [42]. A smaller size of $210 \mu \mathrm{m} \times 210 \mu \mathrm{m}$ is achieved based on photonic crystal slabs with a $1.5 \mathrm{~nm}$ spectral resolution and a $200 \mathrm{~nm}$ system bandwidth [43]. The quantum dot spectrometer that uses CQDs as broadband filter has a spectral resolution of $3.2 \mathrm{~nm}$ and a system bandwidth of $300 \mathrm{~nm}$ with the unit size of $8.5 \mathrm{~mm} \times 6.8 \mathrm{~mm}$ [40]. At present, the record of the smallest spectrometer is reported by the single nanowire spectrometer. By using nanowire, a spectral resolution of $10 \mathrm{~nm}$ and a system bandwidth of $130 \mathrm{~nm}$ can be achieved with the unit size of $0.5 \mu \mathrm{m} \times 75 \mu \mathrm{m}$ [41].

\section{Temporal speckle-based spectrum analyzer (TSSA)}

For TSSA, the most significant difference from SSSA is that only a single PD is needed to receive the speckle. System simplification, cost reduction, and potentially fast measurement speed are the advantages of using a single PD. For current TSSA implementations, Rayleigh speckle is obtained from an SMF for spectrum measurement. Rayleigh scattering is an optical phenomenon that the lightwave is elastically scattered by particles much smaller than the lightwave wavelength [61], which can be observed in SMF [62]. Since the scattered lightwave is constrained to propagate along the fiber, it is feasible to detect the Rayleigh backscattering (RBS) lightwave via a fiber circulator. There are many implementations to utilize RBS lightwave for distributed sensing [63] and fiber link monitoring [64]. It is noticed that the refractive-index inhomogeneities in the optical fiber are inevitable as the fabrication process can not be perfect. Owing to the inhomogenous refractive-index, RBS lightwave from different reflection points along the fiber with random phases interferes with each other, and then produces the Rayleigh speckle with a jagged appearance [65]. This jagged appearance, also named as fading noise, is undesirable for measurements in the field of distributed sensing and many efforts have been made to eliminate it $[66,31]$. However, this unique jagged speckle which contains the spectrum information of the incident lightwave can be used to determine the wavelength. We recently proposed two types of Rayleigh-speckle based wavemeter based on the characteristics of Rayleigh speckle varying with wavelength. According to the different modulation methods of the incident lightwave, it can be divided into pulse modulation Rayleigh speckle wavemeter (PMRSW) and chirp modulation Rayleigh speckle wavemeter (CMRSW).

\subsection{Principle and configuration}

The schematic of the proposed PMRSW is shown in Fig.3(a). After the lightwave is launched to the system, it is modulated by an acousto-optic modulator (AOM) to produce pulsed lightwave for the detection by optical time domain reflectometry (OTDR). The pulsed lightwave is injected into the SMF which is set in a sealed box to eliminate environmental perturbation via the port 1 of the fiber circulator. Using one-dimensional discrete model of 


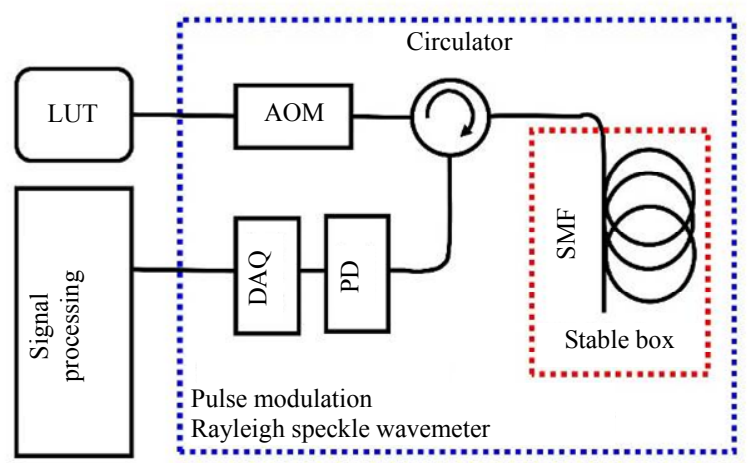

(a)

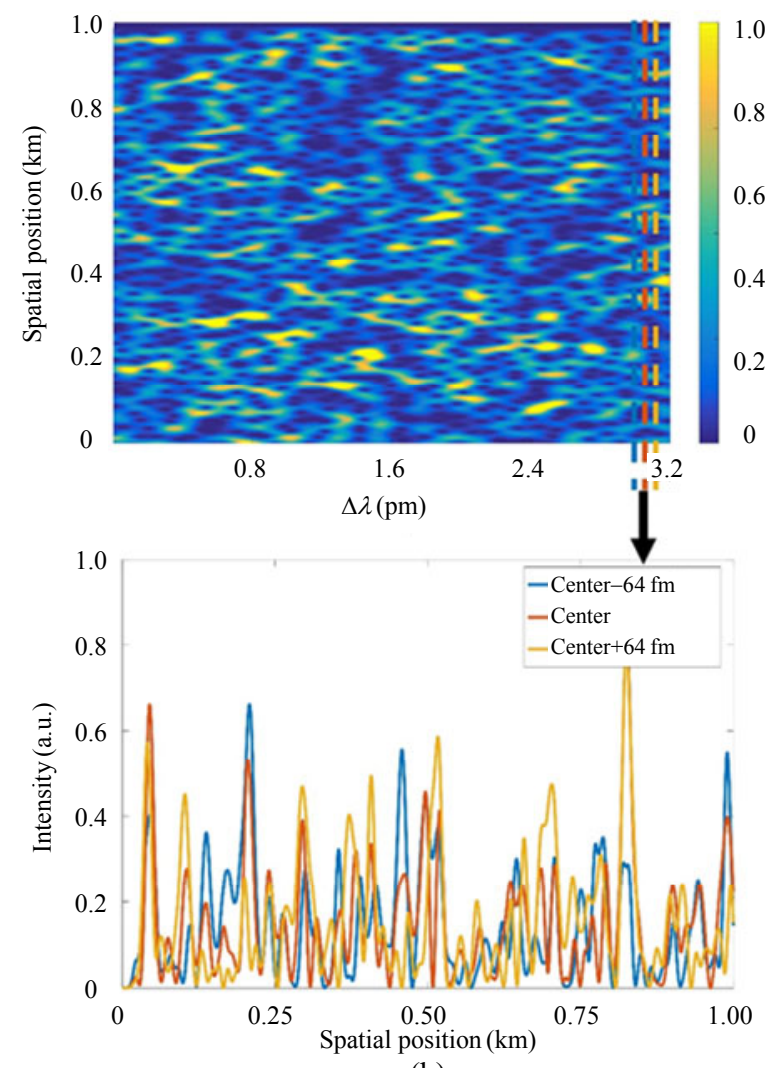

(b)

Fig. 3 Schematic and principle of pulse modulation Rayleigh speckle wavemeter: (a) experimental setup of pulse modulation Rayleigh speckle wavemeter. LUT, lightwave under test; AOM, acousto-optic modulator; PD, photodetector; DAQ, data acquisition; SMF, single mode fiber; (b) Rayleigh speckle patterns at different wavelengths for a probe pulse of $200 \mathrm{~ns}$ width.

fiber-optics to analyze RBS lightwave is a simple, effective, and acceptable method [65]. Therefore, RBS lightwave at position $a$ of fiber can be written as

$$
E_{a}=\sum_{m=a}^{a+N} A_{m} \exp \left\{\mathrm{j} 2 \pi f t-\mathrm{j} \int_{0}^{z_{m}} \frac{2 \pi n(z)}{\lambda} \mathrm{d} z+\mathrm{j} \varphi_{0}\right\}
$$

where $A_{m}=\mathrm{A} \exp \left(-\alpha z_{m}\right), \varphi_{0}$ is the initial phase, $c$ is the lightwave velocity, $n(z)$ represents the refractive index at position $z_{a}, \alpha$ is the attenuation coefficient of fiber, $f$ and $\lambda$ are the optical frequency and wavelength, respectively. At Port 3 of the fiber circulator, the intensity received by the PD is formed by the interference between the RBS lightwave at different positions on the optical fiber within a pulse. The number of scattering points $N$ involved in the interference is roughly equal to $W_{p} v / \lambda$, where $W_{p}$ is the pulse width and $v$ is lightwave velocity in the fiber. The detected intensity of Rayleigh speckle at $z_{a}$ position can be expressed as

$$
I_{a} \propto 2 \sum_{m=a}^{a+N-1} \sum_{n=a}^{a+N-1} A_{m} A_{n} \exp \left\{\mathrm{j} \frac{2 \pi}{\lambda} \int_{-z_{n}}^{z_{m}} n(z) \mathrm{d} z\right\} .
$$

From (6), it is obvious that the detected speckle contains the wavelength information of the incident lightwave. Figure 3(b) reveals the characteristics that the Rayleigh speckle patterns are varying with different wavelengths. Same as SSSA, the transmission matrix method is chosen as the spectrum recovery algorithm for PMRSW.

Figure 4(a) shows the schematic set-up of CMRSW, which includes three parts: a single side-band (SSB) modulator, a fiber interferometer, and a balanced photodetector (BPD). The SSB modulator is used to modulate the lightwave as a frequency swept signal with a swept range of $f_{s}$, a linear slope of $\gamma$, and a central frequency of $f_{u}$. Before the chirped lightwave is injected into the interferometer, the polarization state is fixed by using a fiber-optic polarizer (FOP). The lightwave is split into two paths by the interferometer, and the modulated lightwave in the lower path enters SMF through the circulator to produce RBS lightwave. The Rayleigh speckle generated by the interference between RBS lightwave and local lightwave is received by BPD and can be expressed as

$$
I(t)=\sum_{i} R_{i} E_{0}^{2} \cos \left[2 \pi\left(f_{u} \tau_{i}+\gamma \tau_{i} t-\frac{1}{2} \gamma \tau_{i}^{2}\right)\right]
$$

where $E_{0}$ is the amplitude of lightwave, and $R_{i}$ 


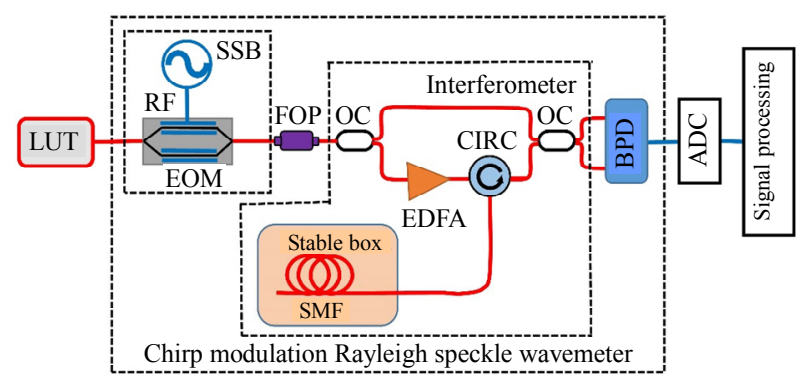

(a)

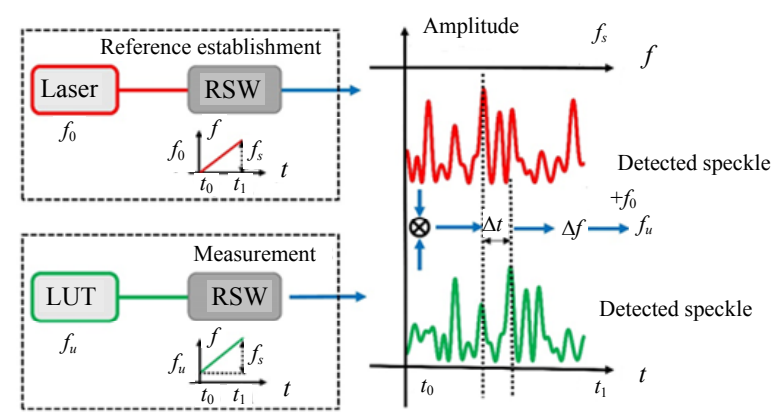

(b)

Fig. 4 Schematic and principle of chirp modulation Rayleigh speckle wavemeter: (a) experimental setup of the chirp modulation Rayleigh speckle wavemeter. LUT, light under test; $\mathrm{EOM}$, electro-optic modulator; RF, radio frequency; SSB, single-sideband; FOP, fiber optic polarizer; OC, optical coupler; EDFA, erbium-doped fiber amplifier; CIRC, circulator; SMF, single mode fiber; BPD, balanced photodetector; ADC, analogto-digital converter; (b) working principle. $f_{0}$, the frequency of laser; $f_{u}$, the frequency of LUT; $f_{s}$, the sweeping range of the modulation; $t_{0}, t_{1}$, the start time and end time of frequency swept modulation, respectively; $\gamma$, the sweeping rate of the modulation; $\Delta f$, the frequency difference between $f_{0}$ and $f_{u}$.

and $\tau_{i}$ are the Rayleigh reflection coefficient and round-trip time of the $i$ th Rayleigh scatter on the fiber, respectively. As expressed by (7), the intensity of Rayleigh speckle varies with time as the phase term is random which is caused by the inhomogeneous distribution of Rayleigh scatter points. Different with PMRSW, the Rayleigh speckle generated in CMRSW has a time-shift characteristic as the wavelength changes. It is obvious from (7) that the change of frequency $\Delta f$ only brings about an extra phase term $2 \pi \Delta f \tau_{i}$ which makes the Rayleigh speckle shift in time domain. This unique characteristic makes the cross-correlation an effective and suitable algorithm for spectrum recovery in the CMRSW and the working principle is illustrated in Fig. 4(b). Before the measurement process, a laser with a determined optical frequency $f_{0}$ is served as the reference light source for reference speckle generation. After receiving the Rayleigh speckle with an unknown frequency of $f_{u}$, the cross-correlation algorithm is adopted to determine $f_{u}$ by the time shift $\Delta t$ between reference speckle and measured speckle. Once the time shift $\Delta t$ is identified by the shift of cross-correlation peak, $f_{u}$ can be calculated via the relation of $f_{u}=f_{0}+\gamma \Delta t$.

\subsection{Spectral resolution}

The spectral resolution of PMRSW is defined by the HWHM of the correlation curve same as the definition for SSSA [67]. For PMRSW, the correlation coefficient as a function of $\Delta \lambda$ is shown in Fig. 5(a). From (6), it is intuitive that Rayleigh speckles with different patterns are produced with different pulse widths. The Rayleigh speckle with a wider pulse width is formed by more RBS lightwave, which makes the pattern of Rayleigh speckle more sensitive to the wavelength changes. Experiments have been implemented to reveal the inverse relation between pulse width and resolution, and the result is shown in Fig. 5(b). The resolution determined by the HWHM of the correlation curve is used for spectrometer and $6 \mathrm{fm}$ resolution is achieved with $2 \mathrm{~ms}$ pulse. By using a correlation coefficient comparison method, the resolution can be further improved for wavemeter applications. Thanks to the stability of correlation curve, it is practicable to determine the wavelength by comparing the correlation coefficients between the measured Rayleigh speckle and the reference speckles. Therefore, the fluctuation degree of the correlation coefficient can be regarded as the accuracy of the method. By using this method, the spectral resolution of $0.4 \mathrm{fm}$ is achieved with a 15 -fold improvement at $2 \mathrm{~ms}$ pulse width. 


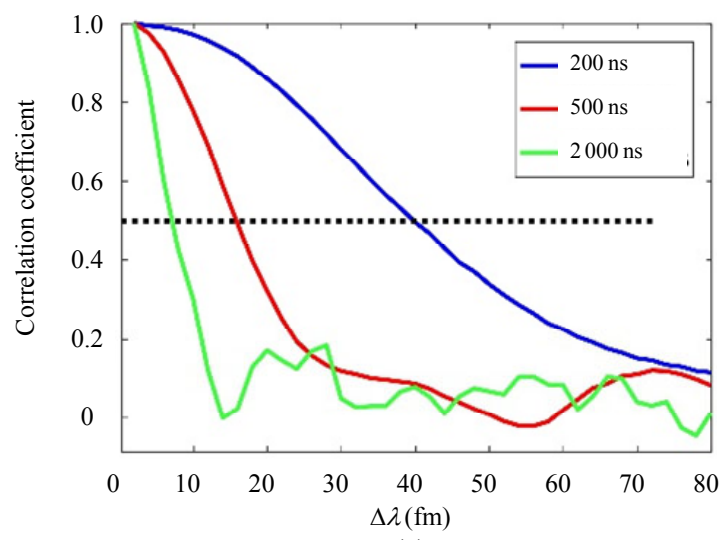

(a)

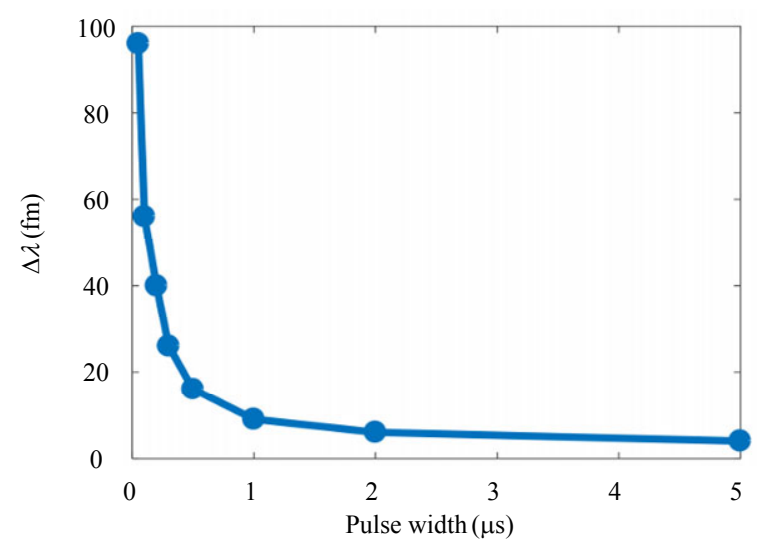

(b)

Fig. 5 Relationship between wavelength resolution and pulse width: (a) correlation coefficient as a function of $\Delta \lambda$ with different pulse widths. Black dotted line corresponds to the correlation coefficient of 0.5 ; (b) the wavelength resolution as a function of pulse width.

Since CMRSW acquires the wavelength information by the shift of cross-correlation peak, the spectral resolution is related to the peak width of the cross-correlation curve [68]. It is clear that a narrower cross correlation peak width is helpful for a more accurate peak location determination and provides a higher resolution. The fiber length $L$ is the major factor that has effect on the peak width $W$ and the relation is demonstrated in the reference as $2 W L=v$. Although the peak width reflects the measurement capability of the system, it is not equal to the system resolution. Here, the resolution is identified as the standard deviation (std.) of the fluctuation when the wavelength is fixed [69]. The statistical results of multiple measurements show that the system has a resolution of 64 am with a $2 \mathrm{~km}$ SMF. However, Rayleigh speckle is susceptible to

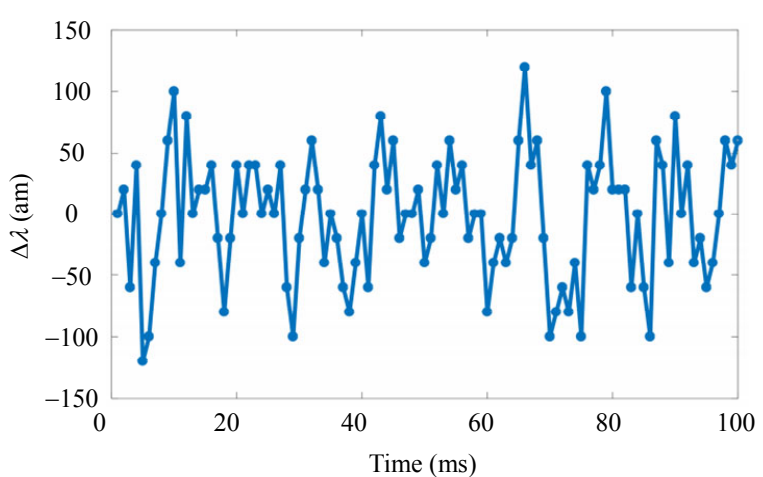

(a)

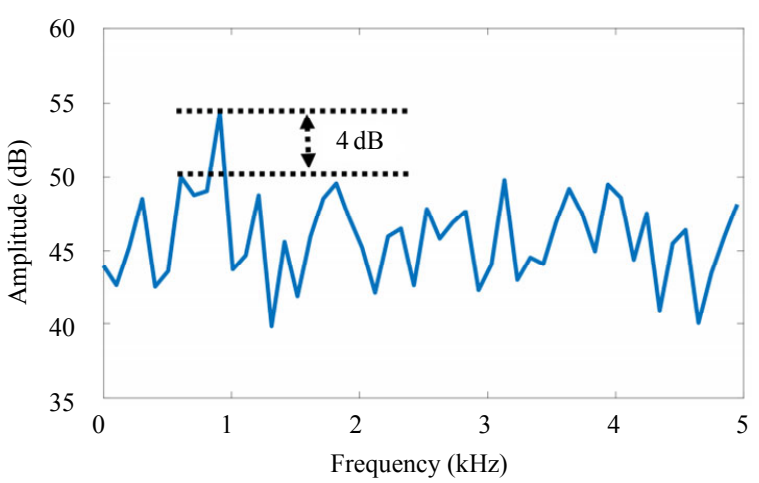

(b)

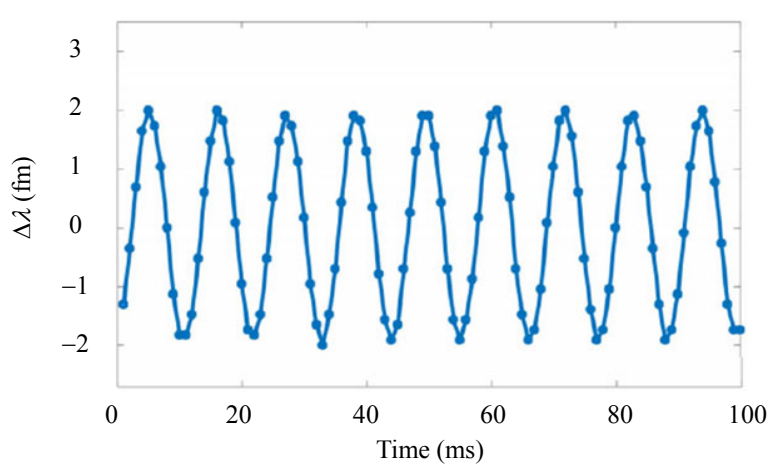

(c)

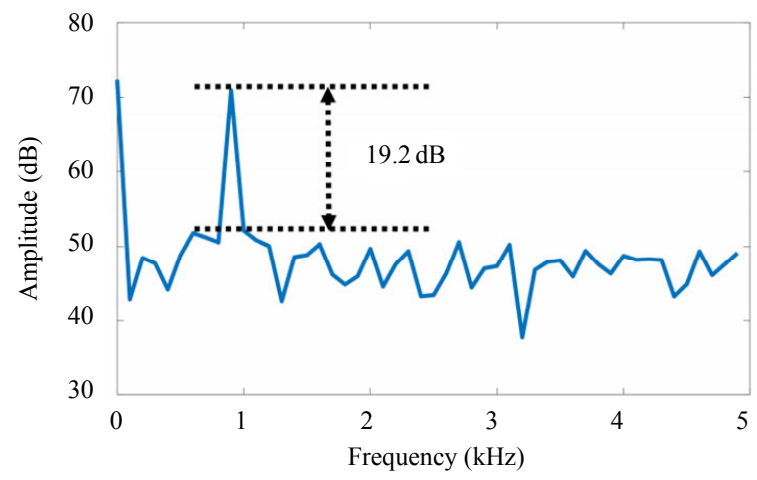

(d)

Fig. 6 Resolution of Rayleigh speckle-based wavemeter (RSW). Wavelength modulation of a stable laser with $900 \mathrm{~Hz}$ frequency and an amplitude of (a) 40 am and (c) $2 \mathrm{fm}$; (b) and (d) are single-sided amplitude spectra of (a) and (c). 
deformation with a long SMF due to the phase noise caused by environmental perturbations. The long fiber is also not conducive to the system compactness. The spectrum refinement method is then introduced to realize a high-resolution system with a short SMF [70]. A sinusoidal wavelength modulated lightwave with an amplitude of $40 \mathrm{am}$ can be measured by applying spectrum refinement method with a $100 \mathrm{~m} \mathrm{SMF}$ and the results are shown in Fig. 6.

\subsection{System bandwidth}

Since the limiting factors of system bandwidth and spectral resolution are independent of each other, there is no trade-off relation between the two parameters in the TSSA. In theory, the TSSA bandwidth is the transmission window of SMF. However, the limitations of commercial equipment make it difficult to experimentally achieve a broad bandwidth measurement with a high resolution.

Since the spectrum reconstruction algorithm of PMRSW is the transmission matrix method, the bandwidth and resolution ultimately depend on the spectral range and the interval of the available reference Rayleigh speckles. Measurement results with $25 \mathrm{~nm}$ bandwidth and $1 \mathrm{~nm}$ resolution are used to demonstrate the broad bandwidth capability of the system [67]. Limited by the wavelength adjustable range of $\mathrm{AOM}$ and the lackness of suitable tunable lasers, the system can only achieve a bandwidth of $168 \mathrm{fm}$ with a resolution of $6 \mathrm{fm}$. For CMRSW, the wavelength is determined by the time shift between the measured speckle and the reference speckle [69]. Therefore, the system bandwidth is actually equal to the frequency range covered by the reference speckle. In experiments, the frequency range of the reference speckle is limited by the swept range $f_{s}$ of the SSB modulator which restricts the system bandwidth. In order to obtain an ultra-wide bandwidth without increasing the system complexity, a spectrum stitching method is proposed [70]. At the stage of obtaining the reference speckle, the lightwave with frequency intervals of $f_{s} / 2$ is injected into the system to generate the corresponding Rayleigh speckles covering a bandwidth of $f_{s}$. By finding the same part of these speckles and stitching them together, we can obtain a reference speckle covering a large bandwidth. The ultra-wide bandwidth of $80 \mathrm{~nm}$ is achieved with the spectrum stitching method. Note that the system does not sacrifice the spectra resolution while achieving such a large bandwidth. It is the first demonstration that a broad bandwidth of $80 \mathrm{~nm}$ and a resolution of sub-femtometer are achieved simultaneously for a wavemeter.

\subsection{Measurement rate}

Compared with SSSA, a fast measurement rate is expected for the temporal speckle-based systems. Since the pulsed lightwave needs to go through a round trip on the SMF to produce Rayleigh speckle, the measurement rate of PMRSW is limited by the fiber length [67]. A $40 \mathrm{kHz}$ measurement rate is achieved with $2 \mathrm{~km}$ SMF. This fast measurement speed is experimentally verified by measuring a multi-wavelength laser source with a wavelength modulation at a time resolution of $25 \mu \mathrm{s}$. Not only the fiber length, but also the frequency sweep time is the limiting factor of the measurement rate for CMRSW. After comprehensively considering the performance of available experimental devices, a measurement rate of $10 \mathrm{kHz}$ is realized [70].

\section{Applications}

There have been some interesting applications based on speckle-based wavemeter. The speckle wavemeter based on integrating sphere is served as a part of feedback loop to provide wavelength information in the laser stablization configuration as shown in Fig. 7 [16]. With this configuration, the linewidth of a $780 \mathrm{~nm}$ diode laser can be achieved better than $1 \mathrm{MHz}$. The update rate of the feedback loop is $200 \mathrm{~Hz}$ limited by the processing speed of PCA algorithm. Poincare analysis can be used to replace PCA algorithm to achieve a real-time $(>4 \mathrm{kHz})$ measurement rate with an fm resolution 
[35]. In addition to stabilize the laser, it can also be used to measure the absorption spectrum by combining the speckle-based wavemeter with a tunable laser, as shown in Fig. 8. The tunable laser performs a wide-range wavelength sweeping, and the wavemeter is responsible for accurately determining the wavelength change during the sweeping. The system can realize a precise and broadband measurement of absorption spectrum due to the high performance of CMRSW. The intensity spectrum of $H 13 C 14 N$ gas covering a bandwidth from $1520 \mathrm{~nm}$ to $1600 \mathrm{~nm}$ is accurately measured with an ultra-high resolution of $40 \mathrm{am}$ by using CMRSW [70].

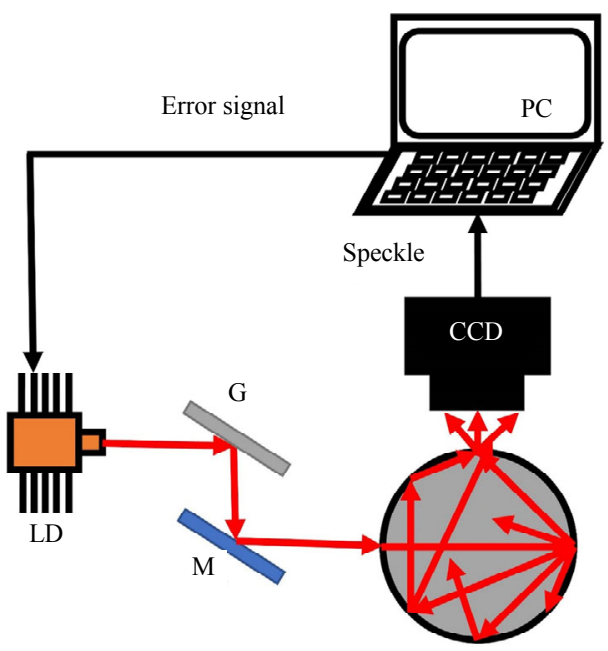

Fig. 7 Experimental setup of the control loop for laser stabilization via speckle-based wavemeter. LD, laser diode; G, diffraction grating; $M$, mirror.

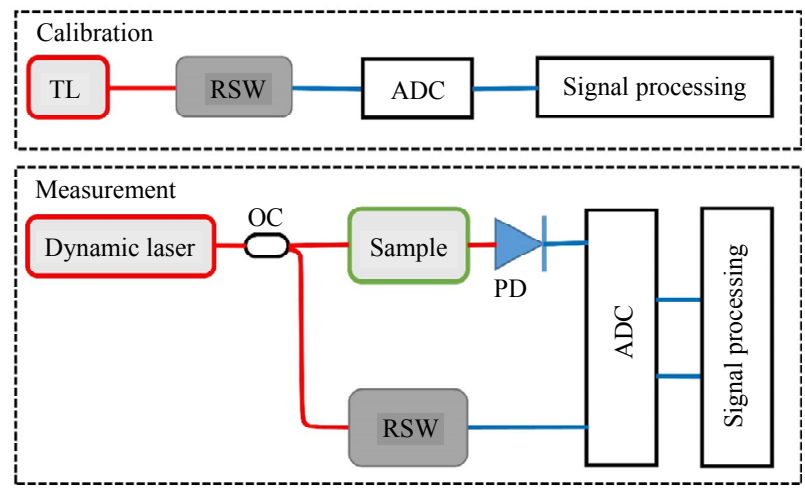

Fig. 8 Experimental setup for RSW to enable spectroscopy measurements. TL, tunable laser; PD, photodetector; ADC, analog-to-digital converter.

For the measurement of broad spectrum, MMF-based spectrometer is the most promising system. A measurement of photoluminescence covering $400 \mathrm{~nm}-750 \mathrm{~nm}$ is implemented by MMF-based spectrometer with the resolution of $1 \mathrm{~nm}$ [47]. The MMF-based spectrometer can be used not only to measure the spectrum with broad bandwidth, but also to detect the spectrum of the optical frequency comb (OFC) [71]. OFC has become a preferred tool and plays an important role in many research fields for its excellent frequency accuracy and broad spectral coverage [72]. In order to make full use of OFC, it is crucial to obtain the spectrum of the OFC. MMF-based spectrometer has been proven to be an effective scheme for direct comb spectroscopy and the scheme setup is shown in Fig. 9. A simultaneous measurement of 500 isolated comb lines with a repetition frequency of $250 \mathrm{MHz}$ is demonstrated. By performing sequential acquisitions, a larger measurement bandwidth over $0.85 \mathrm{THz}$ is also achieved [71].

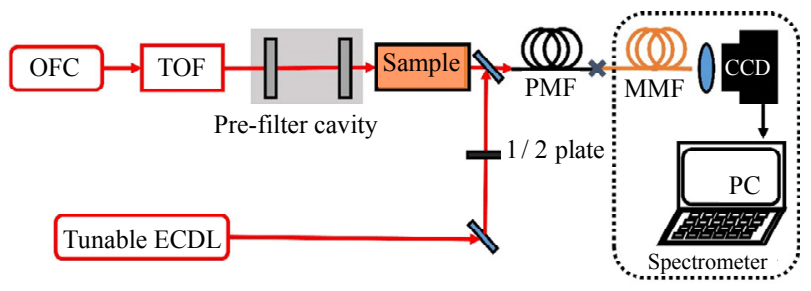

Fig. 9 Experimental setup for parallel comb spectroscopy by using MMF-based spectrometer. OFC, optical frequency comb; TOF, tunable optical filter; PMF, polarization-maintaining fiber; MMF, multi-mode fiber; PC, personal computer; ECDL, extended cavity diode laser.

Due to the advantages of high-performance and compactness, the speckle-based spectrometers are also introduced into the fields of optical coherence tomography [18] and hyperspectral imaging [38, 73] for precise measurement.

\section{Discussion}

Although the speckle-based spectrum analyzers can provide measurement with broad-bandwidth and high-resolution, there are still many challenges which hinder the commercialization and are needed to overcome.

\subsection{Stabilization}

The major open challenge for all types of 
speckle-based spectrum analyzers is to keep the system stable [27]. The speckle is sensitive to environmental perturbation, especially for the high-resolution applications. How to isolate the environment influence on the speckle to maintain the system accuracy is an important and tough task. A solution combining thermal and mechanical stabilization with the software corrections is applied in the MMF-based spectrometer [71]. After adopting this simple correction method, the measurement error is reduced from $\pm 15 \mathrm{pm}$ to $\pm 3 \mathrm{pm}$ over 10 hours [47]. To ensure the accuracy of measurement results, a multi-layer temperature-stable box with a temperature control system is designed for the stabilization in Rayleigh speckle-based wavemeter [70]. The experimental results show that the measurement error of the system within 1 hour is $3.1 \mathrm{fm}$. It is believed that the stable box can be further improved by a careful mechanical and temperature stabilization engineering to provide a better accuracy for the speckle-based spectrum analyzers. It is worth noting that the on-chip speckle-based spectrometer has low requirements for environmental stability due to its small size and relatively low resolution. An adaptive thermal stabilization method is proposed by extracting the fringe drift for thermal stabilization in the integrated waveguide spectrometer, which is meaningful for the economical and robust on-chip spectrometer [74].

\subsection{Noise analysis}

The noise characteristics of the speckle-based spectrum analyzer are also necessary to be discussed in detail. By determining the noise sources, researchers can identify the allowable minimum power of incident lightwave for the system. A comprehensive analysis of the noise effect on the MMF-based spectrometer has been given [46]. The comparison experiment results show that the MMF-based spectrometer has a comparable sensitivity to the conventional grating-based spectrometer. The influence of the noise on PMRSW is also investigated. Both simulation and experiment are performed to study the influence of the main noise, i.e. shot noise and thermal noise, on the spectrum recovery results [67].

\subsection{Optical efficiency}

Optical efficiency is defined as the ratio of detected photons to incident photons. The spectrum analyzer with a high optical efficiency has advantages in detecting weak lightwave. In the speckle-based spectrometers or wavemeters, the lightwave is passed through a random medium and only a part of the scattered lightwave is detected which results in a low optical efficiency. It is demonstrated that a scattering metal waveguide can be utilized along with a prism speckle spectrometer to improve the optical efficiency by two orders of magnitude, from $0.01 \%$ to $1.3 \%$ [19]. Moreover, an inverse design method is proposed to design the scattering medium in order to maximize spectral response for on-chip spectrometry [75]. Some methods may be used to improve optical efficiency for other systems. Optimizing the tapering parameters of the fiber or depositing nanoscatterers may enhance the detection for multimode taper-based spectrometer [20]. As for the Rayleigh speckle wavemeter, Rayleigh scattering process enhancement methods, such as ultraviolet exposure [76] and weak reflection point writing [77], can be considered to be applied for the improvement of optical efficiency.

\section{Conclusions}

In this review, we introduce the principles of different types of speckle-based spectrum analyzer and describe an overview of recent advances in the improvement of system bandwidth, spectral resolution, and measurement rate. We then discuss the open challenges in this field. Moreover, applications realized by utilizing the highperformance speckle-based spectrum analyzer are discussed.

Recent research results show that the 
speckle-based spectrum analyzer has potentials for replacing conventional spectrometers with advantages of high-resolution, broad-bandwidth, and compact size. Some works have indicated that it is feasible to integrate a stable and high-performance speckle-based spectrometer on chip. However, the largest obstacle to commercialization is the system long-term stability, which should be the main focus of the future research. After solving the challenge of stability, the speckle-based spectrum analyzer with high-performance will become a prevailing instrument which brings breakthroughs and new discoveries in many fields, such as spectroscopy, sensing, communication, and imaging.

\section{Acknowledgment}

This work was supported by the National Natural Science Foundation of China (NSFC) (Grant Nos. 61775132, 61735015, and 61620106015).

Open Access This article is distributed under the terms of the Creative Commons Attribution 4.0 International License (http://creativecommons.org/licenses/by/4.0/), which permits unrestricted use, distribution, and reproduction in any medium, provided you give appropriate credit to the original author(s) and the source, provide a link to the Creative Commons license, and indicate if changes were made.

\section{References}

[1] K. A. Willets and R. P. Van Duyne, "Localized surface plasmon resonance spectroscopy and sensing," Annual Review of Physical Chemistry, 2007, 58: 267-297.

[2] P. A. Martin, "Near-infrared diode laser spectroscopy in chemical process and environmental air monitoring," Chemical Society Reviews, 2002, 31(4): 201-210.

[3] R. A. Halvorson and P. J. Vikesland, "Surface-enhanced Raman spectroscopy (SERS) for environmental analyses," Environmental Science \& Technology, 2010, 44(20): 7749-7755.

[4] C. Kulesa, "Terahertz spectroscopy for astronomy: From comets to cosmology," IEEE Transactions on Terahertz Science and Technology, 2011, 1(1): 232-240.

[5] F. Hoyle, N. Wickramasinghe, S. Al-Mufti, A. Olavesen, and D. Wickramasinghe, "Infrared spectroscopy over the 2.9-3.9 $\mu \mathrm{m}$ waveband in biochemistry and astronomy," in Astronomical Origins of Life, Springer, 2000, pp. 161-166,

[6] L. P. Choo-Smith, H. Edwards, H. P. Endtz, J. Kros, F. Heule, H. Barr, et al., "Medical applications of Raman spectroscopy: from proof of principle to clinical implementation," Biopolymers: Original Research on Biomolecules, 2002, 67(1): 1-9.

[7] A. Sakudo, "Near-infrared spectroscopy for medical applications: current status and future perspectives," Clinica Chimica Acta, 2016, 455: 181-188.

[8] T. Matsumoto, S. Fujita, and T. Baba, "Wavelength demultiplexer consisting of photonic crystal superprism and superlens," Optics Express, 2005, 13(26): 10768-10776.

[9] A. T. U. R. O. AP2041B. Available at http://www.apext.com/pdf/optical-spectrum-analyzer .pdf.

[10] Y. O. AQ6370D. Available at https://www.yokogawa.com/pdf.

[11] P. R. Griffiths and J. A. De Haseth, "Fourier transform infrared spectrometry," New Jersey: John Wiley \& Sons, 2007: 171.

[12] D. M. Kita, B. Miranda, D. Favela, D. Bono, J. Michon, H. Lin, et al., "High-performance and scalable on-chip digital Fourier transform spectroscopy," Nature Communications, 2018, 9(1): $1-7$.

[13] M. C. Souza, A. Grieco, N. C. Frateschi, and Y. Fainman, "Fourier transform spectrometer on silicon with thermo-optic non-linearity and dispersion correction," Nature Communications, 2018, 9(1): $1-8$.

[14] Q. Cheng, F. Duan, T. Huang, and J. Wang, "Forward fiber Fourier transform spectrometer modeling and design with PZT phase modulation real-time compensation," Applied Optics, 2018, 57(18): 5025-5035.

[15] M. Chakrabarti, M. L. Jakobsen, and S. G. Hanson, "Speckle-based spectrometer," Optics Letters, 2015, 40(14): 3264-3267.

[16]N. K. Metzger, R. Spesyvtsev, G. D. Bruce, B. Miller, G. T. Maker, G. Malcolm, et al., "Harnessing speckle for a sub-femtometre resolved broadband wavemeter and laser stabilization," Nature Communications, 2017, 8: 15610.

[17] B. Redding and H. Cao, "Using a multimode fiber as a high-resolution, low-loss spectrometer," Optics Letters, 2012, 37(16): 3384-3386.

[18] Ş. K. Çetindağ, M. F. Toy, O. Ferhanoğlu, and F. Çivitci, "A speckle-enhanced prism spectrometer with high dynamic range," IEEE Photonics Technology Letters, 2018, 30(24): 2139-2142.

[19] S. K. Çetindağ, M. F. Toy, O. Ferhanoğlu, and F. Civitci, "Scattering metal waveguide based speckle-enhanced prism spectrometry," Journal of Lightwave Technology, 2020, 38(7): 2022-2027.

[20] N. H. Wan, F. Meng, T. Schröder, R.-J. Shiue, E. H. Chen, and D. Englund, "High-resolution optical 
spectroscopy using multimode interference in a compact tapered fibre," Nature Communications, 2015, 6(1): 1-6.

[21] A. Shamsoddini and J. C. Trinder, "Image texture preservation in speckle noise suppression," ISPRS TC VII Symposium - 100 Years ISPRS, 2010, 7: 239-244.

[22] W. S. Ha, S. J. Lee, K. H. Oh, Y. M. Jung, and J. K. Kim, "Speckle reduction in near-field image of multimode fiber with a piezoelectric transducer," Journal of the Optical Society of Korea, 2008, 12(3): 126-130.

[23] E. Fujiwara, M. F. M. dos Santos, and C. K. Suzuki, "Optical fiber specklegram sensor analysis by speckle pattern division," Applied Optics, 2017, 56(6): 1585-1590.

[24]E. Fujiwara, L. E. da Silva, T. H. Marques, and C. M. Cordeiro, "Polymer optical fiber specklegram strain sensor with extended dynamic range," Optical Engineering, 2018, 57(11): 116107.

[25] P. Wu, S. Zhu, M. Hong, F. Chen, and H. Liu, "Specklegram temperature sensor based on femtosecond laser inscribed depressed cladding waveguides in Nd: YAG crystal," Optics \& Laser Technology, 2019, 113: 11-14.

[26] Y. Liu, Q. Qin, H. H. Liu, Z. W. Tan, and M. G. Wang, "Investigation of an image processing method of step-index multimode fiber specklegram and its application on lateral displacement sensing," Optical Fiber Technology, 2018, 46: 48-53.

[27] H. Cao, "Perspective on speckle spectrometers," Journal of Optics, 2017, 19: 060402.

[28] B. Redding, S. F. Liew, R. Sarma, and H. Cao, "Compact spectrometer based on a disordered photonic chip," Nature Photonics, 2013, 7(9): $746-751$.

[29] P. Gysel and R. K. Staubli, "Statistical properties of Rayleigh backscattering in single-mode fibers," Journal of Lightwave Technology, 1990, 8(4): 561-567.

[30] P. Healey, "Fading in heterodyne OTDR," Electronics Letters, 1984, 20(1): 30-32.

[31] K. Shimizu, T. Horiguchi, and Y. Koyamada, "Characteristics and reduction of coherent fading noise in Rayleigh backscattering measurement for optical fibers and components," Journal of Lightwave Technology, 1992, 10(7): 982-987.

[32] J. Zhou, Z. Pan, Q. Ye, H. Cai, R. Qu, and Z. Fang, "Characteristics and explanations of interference fading of a phi-OTDR with a multi-frequency source," Journal of Lightwave Technology, 2013, 31(17): 2947-2954.

[33] B. Redding, S. M. Popoff, and H. Cao, "All-fiber spectrometer based on speckle pattern reconstruction," Optics Express, 2013, 21(5): 6584-6600.

[34] S. G. Hanson, M. L. Jakobsen, and M. Chakrabarti, "The dynamic speckle-based wavemeter," in
SPECKLE 2018: VII International Conference on Speckle Metrology, Poland, 2018, pp: 10834: 108342D.

[35] L. ODonnell, K. Dholakia, and G. D. Bruce, "High speed determination of laser wavelength using Poincaré descriptors of speckle," Optics Communications, 2020, 459: 124906.

[36] A. Dávila and J. Rayas, "Single-shot phase detection in a speckle wavemeter for the measurement of femtometric wavelength change," Optics and Lasers in Engineering, 2020, 125: 105856.

[37]Y. Kwak, S. M. Park, Z. Ku, A. Urbas, and Y. L. Kim, "A pearl spectrometer," Nano Letters, 2021, 21(2): 921-930.

[38] K. Monakhova, K. Yanny, N. Aggarwal, and L. Waller, "Spectral diffusercam: lensless snapshot hyperspectral imaging with a spectral filter array," Optica, 2020, 7(10): 1298-1307.

[39] E. Huang, Q. Ma, and Z. Liu, "Etalon array reconstructive spectrometry," Scientific Reports, 2017, 7(1): 1-6.

[40] J. Bao and M. G. Bawendi, "A colloidal quantum dot spectrometer," Nature, 2015, 523(7558): 67-70.

[41] Z. Yang, T. Albrow-Owen, H. Cui, J. Alexander-Webber, F. Gu, X. Wang, et al., "Single-nanowire spectrometers," Science, 2019, 365(6457): 1017-1020.

[42] X. Gan, N. Pervez, I. Kymissis, F. Hatami, and D. Englund, "A high-resolution spectrometer based on a compact planar two-dimensional photonic crystal cavity array," Applied Physics Letters, 2012, 100(23): 231104.

[43] Z. Wang, S. Yi, A. Chen, M. Zhou, T. S. Luk, A. James, et al., "Single-shot on-chip spectral sensors based on photonic crystal slabs," Nature Communications, 2019, 10(1): 1-6.

[44] C. Kim, W. B. Lee, S. K. Lee, Y. T. Lee, and H. =N. Lee, "Fabrication of 2D thin-film filter-array for compressive sensing spectroscopy," Optics and Lasers in Engineering, 2019, 115: 53-58.

[45] T. W. Kohlgraf-Owens and A. Dogariu, "Transmission matrices of random media: means for spectral polarimetric measurements," Optics Letters, 2010, 35(13): 2236-2238.

[46] B. Redding, S. M. Popoff, Y. Bromberg, M. A. Choma, and H. Cao, "Noise analysis of spectrometers based on speckle pattern reconstruction," Applied Optics, 2014, 53(3): 410-417.

[47] B. Redding, M. Alam, M. Seifert, and H. Cao, "High-resolution and broadband all-fiber spectrometers," Optica, 2014, 1(3): 175-180.

[48] T. Wang, Y. Li, Y. Meng, Y. Qiu, and B. Mao, "Study of a fiber spectrometer based on offset fusion," Applied Optics, 2020, 59(15): 4697-4702.

[49] T. Wang, Y. Li, B. Xu, B. Mao, Y. Qiu, and Y. Meng, "High-resolution wavemeter based on polarization modulation of fiber speckles," APL Photonics, 2020, 
5(12): 126101

[50] G. D. Bruce, L. ODonnell, M. Chen, and K. Dholakia, "Overcoming the speckle correlation limit to achieve a fiber wavemeter with attometer resolution," Optics Letters, 2019, 44(6): 1367-1370.

[51] G. D. Bruce, L. ODonnell, M. Chen, M. Facchin, and K. Dholakia, "Femtometer-resolved simultaneous measurement of multiple laser wavelengths in a speckle wavemeter," Optics Letters, 2020, 45(7): 1926-1929.

[52] R. K. Gupta, G. D. Bruce, S. J. Powis, and K. Dholakia, "Deep learning enabled laser speckle wavemeter with a high dynamic range," Laser \& Photonics Reviews, 2020, 14(9): 2000120.

[53] M. Piels and D. Zibar, "Compact silicon multimode waveguide spectrometer with enhanced bandwidth," Scientific Reports, 2017, 7: 43454.

[54] D. Yi, Y. Zhang, X. Wu, and H. K. Tsang, "Integrated multimode waveguide with photonic lantern for speckle spectroscopy," IEEE Journal of Quantum Electronics, 2020, 57(1): 1-8.

[55] B. Redding, S. F. Liew, Y. Bromberg, R. Sarma, and H. Cao, "Evanescently coupled multimode spiral spectrometer," Optica, 2016, 3(9): 956-962.

[56] S. F. Liew, B. Redding, M. A. Choma, H. D. Tagare, and H. Cao, "Broadband multimode fiber spectrometer," Optics Letters, 2016, 41(9): 2029-2032.

[57] Z. Meng, J. Li, C. Yin, T. Zhang, Z. Yu, M. Tang, et al., "Multimode fiber spectrometer with scalable bandwidth using space-division multiplexing," AIP Advances, 2019, 9(1): 015004.

[58] P. Varytis, D. N. Huynh, W. Hartmann, W. Pernice, and K. Busch, "Design study of random spectrometers for applications at optical frequencies," Optics Letters, 2018, 43(13): 3180-3183.

[59] W. Hartmann, P. Varytis, H. Gehring, N. Walter, F. Beutel, K. Busch, et al., "Waveguide-integrated broadband spectrometer based on tailored disorder," Advanced Optical Materials, 2020, 8(6): 1901602.

[60] W. Hartmann, P. Varytis, H. Gehring, N. Walter, F. Beutel, K. Busch, et al., "Broadband spectrometer with single-photon sensitivity exploiting tailored disorder," Nano Letters, 2020, 20(4): 2625-2631.

[61] A. T. Young, "Rayleigh scattering," Applied Optics, 1981, 20(4): 533-535.

[62] M. Nakazawa, "Rayleigh backscattering theory for single-mode optical fibers," Journal of the Optical Society of America, 1983, 73(9): 1175-1180.

[63] L. Palmieri and L. Schenato, "Distributed optical fiber sensing based on Rayleigh scattering," The Open Optics Journal, 2013, 7(1): 104-127.

[64] Y. Koshikiya, X. Fan, and F. Ito, "Long range and $\mathrm{cm}-$ level spatial resolution measurement using coherent optical frequency domain reflectometry with SSB-SC modulator and narrow linewidth fiber laser," Journal of Lightwave Technology, 2008, 26(18): 3287-3294.
[65] M. D. Mermelstein, R. Posey, G. A. Johnson, and S. T. Vohra, "Rayleigh scattering optical frequency correlation in a single-mode optical fiber," Optics Letters, 2001, 26(2): 58-60.

[66] D. Chen, Q. Liu, and Z. He, "Phase-detection distributed fiber-optic vibration sensor without fading-noise based on time-gated digital OFDR," Optics Express, 2017, 25(7): 8315-8325.

[67] Y. Wan, S. Wang, X. Fan, Z. Zhang, and Z. He, "High-resolution wavemeter using Rayleigh speckle obtained by optical time domain reflectometry," Optics Letters, 2020, 45(4): 799-802.

[68] S. Wang, Z. Zhang, X. Fan, B. Wang, and Z. He, "Calibration-free wavelength measurement with sub-femtometer resolution based on all-fiber Rayleigh speckles," in 2019 Conference on Lasers and Electro-Optics (CLEO), USA, May 5-10, 2019, pp: $1-2$.

[69] Z. Zhang, X. Fan, S. Wang, S. Zhao, B. Wang, Y. Wan, et al., "A novel wavemeter with 64 attometer spectral resolution based on Rayleigh speckle obtained from single-mode fiber," Journal of Lightwave Technology, 2020, 38(16): 4548-4554.

[70] Y. Wan, X. Fan, S. Wang, Z. Zhang, S. Zhao, and Z. $\mathrm{He}$, "Wavemeter capable of simultaneously achieving ultra-high resolution and broad bandwidth by using Rayleigh speckle from single mode fiber," Journal of Lightwave Technology, 2020, 39(7): 2223-2229

[71] N. Coluccelli, M. Cassinerio, B. Redding, H. Cao, P. Laporta, and G. Galzerano, "The optical frequency comb fibre spectrometer," Nature Communications, 2016, 7(1): 1-11.

[72] J. Ye and S. T. Cundiff, "Femtosecond optical frequency comb: principle, operation and applications," Berlin: Springer Science \& Business Media, 2005.

[73] R. French, S. Gigan, and O. L. Muskens, "Speckle-based hyperspectral imaging combining multiple scattering and compressive sensing in nanowire mats," Optics Letters, 2017, 42(9): $1820-1823$.

[74] C. C. Teng, C. Xiong, E. J. Zhang, W. M. Green, and G. Wysocki, "Adaptive thermal stabilization of an integrated photonic spectrometer using parasitic interference fringes," Optics Letters, 2020, 45(12): 3252-3255.

[75] T. Liu and A. Fiore, "Designing open channels in random scattering media for on-chip spectrometers," Optica, 2020, 7(8): 934-939.

[76] S. Loranger, M. Gagné, V. Lambin-Iezzi, and R. Kashyap, "Rayleigh scatter based order of magnitude increase in distributed temperature and strain sensing by simple UV exposure of optical fibre," Scientific Reports, 2015, 5: 11177.

[77] M. Wu, X. Fan, Q. Liu, and Z. He, "Highly sensitive quasi-distributed fiber-optic acoustic sensing system by interrogating a weak reflector array," Optics Letters, 2018, 43(15): 3594-3597. 